\title{
Entwicklung von Sozialarbeit im russischen Justizvollzug
}

Wolfgang Gottschalk

\section{Bericht über die Zusammenarbeit der Vollzugsverwaltungen des Gebietes Archangelsk und des Landes Schleswig-Holstein in den Jahren 2002 und 2003}

\section{Vorgeschichte}

$\mathrm{N}$ ach dem Ende der Sowjetunion begannen die Bemühungen seitens des russischen Justizministeriums in Moskau und der Verantwortlichen in der regionalen Justizverwaltung des Gebietes Archangelsk, einen an problemlösender Sozialarbeit orientierten Vollzug zu gestalten, zunehmend schärfere Konturen zu erhalten: die Zentralverwaltung hat hierzu konkrete Richtlinien erlassen, die westeuropäische Standards der Gefangenenarbeit anstreben. Die Vollzugsverwaltung des Gebietes Archangelsk hatte schon während der letzten 5 Jahre mit mehreren Delegationen, die Schleswig-Holstein besucht haben, und durch Delegationen aus SchleswigHolstein, die sich in Archangelsk informiert haben, die Voraussetzungen für eine innovative Zusammenarbeit auf der fachlichen Ebene geschaffen. Ermöglicht wurde dies zunächst durch die Finanzierung im Rahmen des Alexander-HerzenProgramms (EU) ${ }^{1}$.

Seit Frühjahr 2002 kann eine ausschließlich den Justizverwaltungen zugute kommende Finanzierung durch den Europarat in Anspruch genommen werden, die zunächst für drei Jahre (2002 - 2004) bewilligt worden ist. Dadurch wird ein intensiverer und systematischerer Austausch von Vollzugspersonal möglich als über die Mittel des AHP, die dadurch sehr begrenzt sind, als sie schwerpunktmäßig eine Hochschulförderung darstellen und darüber hinaus auch andere Fachgebiete durch dieses Programm gefördert werden.

\section{Aktivitäten im Jahr 2002}

a) Vom 26. Mai bis 02. Juni 2002 besuchte eine Delegation aus Archangelsk Schleswig-Holstein. ${ }^{2}$ Die Delegationsmitglieder bekamen - wie andere vor ihnen auch einen umfassenden Einblick in die schleswig-holsteinische Vollzugspraxis und besuchten die Justizvollzugsanstalten Neumünster und Lübeck, die Jugendanstalt Schleswig und die Justizvollzugsschule Schleswig.

Der Unterschied zu vorangegangenen Delegationen bestand jedoch darin, daß diese Begegnung mit der für die zunächst drei durch den Europarat finanzierten Jahre einvernehmlich festgelegten Schwerpunktsetzung »Sozialarbeit im Justizvollzug « stattfand und in Verabredungen mündete, künftig das gesamte Austauschprogramm stärker einrichtungsbezogen zu gestalten, um konkrete Szenarien für Sozialarbeit - vornehmlich in russischen Einrichtungen - entwickeln und erproben zu können.

Die Partnerschaft besteht zwischen den folgenden Einrichtungen:

- Anstalt 42/1 in Archangelsk - JVA Neumünster /JVA Lübeck

- Jugendkolonie Archangelsk - Jugendanstalt Schleswig

- Ausbildungszentrum Archangelsk - Justizvollzugsschule Schleswig

Hinsichtlich der letztgenannten Partnerschaft kommt es darauf an, Aus- und Fortbildungsinhalte und -methoden im Kontext zu künftigen Sozialarbeitsmodellen in den anderen beiden Einrichtungen zu entwickeln. b) Der Gegenbesuch der Delegation aus Schleswig-Holstein vom 8. - 14. September $2002^{3}$ baute auf den Ergebnissen der Zusammenarbeit im Mai auf und zeichnete sich durch zweierlei Merkmale aus, die dem Erfordernis kontinuierlicher einrichtungsbezogener Entwicklungsarbeit Rechnung trugen:

- Die Delegation bestand ausschließlich aus Mitarbeiterinnen und Mitarbeitern der o.g. schleswig-holsteinischen Vollzugseinrichtungen und zwar aus allen dort vorhandenen Laufbahngruppen und Funktionsebenen.

- Es fanden erstmalig in den o.g. russischen Einrichtungen am Leitthema orientierte Workshops (schwerpunktmäßig rechts- und tatsachenvergleichenden Inhalts) statt.

Schon im Jahr 2002 stellte es sich als schwierig heraus, die vereinbarte projektbezogene Kommunikation zwischen den Delegationstreffen herzustellen. Immer wieder wurde versucht diese zwischen den Partnereinrichtungen, über e-mail und Dolmetscherinnen in Archangelsk, die nicht Mitarbeiterinnen der Vollzugsverwaltung sind, aufzubauen, meist jedoch nicht mit dem gewünschten Erfolg (vgl. dazu unten Ziff. 4).

c) Außerhalb des Programms, das durch den Europarat finanziert wird und die oben beschriebene themenbezogene Entwicklungsarbeit zum Gegenstand hat, brachte eine eigens hierfür zusammengestellte fünfköpfige Delegation, zu der neben dem damaligen Staatssekretär im Justizministerium Mitarbeiter aus dieser Behörde und aus Vollzugseinrichtungen (JVA Neumünster und Justizvollzugsschule) gehörten, zwei Kleinbusse (ehema- lige Gefangenentransportfahrzeuge), gefüllt mit ausgesondertem aber gut verwendbarem technischem Gerät, nach Archangelsk. Die Reise dauerte vom 1. - 8. September und führte über Rostock - Helsinki (Fähre) nach St. Petersburg und von dort nach Archangelsk. Von der russischen Grenze bei Viborg wurde die Gruppe durch Mitarbeiter der Vollzugsverwaltung des Gebietes Archangelsk eskortiert. Die Finanzierung der Reise erfolgte privat und durch Spenden. Wie auch in Berichten vergleichbarer Unternehmungen (z.B. NRW - Wladimir, Wohlfahrtsverbände aus Schleswig-Holstein) zu lesen ist, bereitete die Freigabe der gespendeten Fahrzeuge und der anderen Gegenstände durch den russischen Zoll erhebliche Schwierigkeiten. Wie uns jetzt berichtet wurde, sind inzwischen alle Spenden (Fahrzeuge und sonstige Sachmittel) an die Vollzugsverwaltung in Archangelsk übergeben worden.

\section{Ergebnisse Ende 2002}

Die zahlreichen Begegnungen zwischen Bediensteten aller Ebenen und Laufbahnen beider Vollzugsverwaltungen hatten bereits in der beschriebenen Zeit vor Beginn des aus Europaratsmitteln geförderten Entwicklungsprogramms (vgl. o. 1.) zu einer Reihe persönlicher Kontakte geführt, deren Fortbestand für den weiteren Verlauf der gemeinsamen Arbeit von Nutzen war und ist. Es würde den Rahmen dieses Berichts sprengen, sie hier alle zu benennen. Exemplarisch seien aber doch die persönliche Verbindung zwischen den Leitern der jeweiligen Bereichsleitungen, General Malov und Dr. Maelicke, genannt, die sich gegenseitig besucht haben und aus eigener Anschauung von der Notwendigkeit eines Projektes dieser Art überzeugt sind. Sie unterstützen und begleiten die weitere Zusammenarbeit in erheblichem Maße. Ähnliches gilt für die Anstalts- und Delegationsleiter, die nun die weitere Arbeit gestalten oder zumindest fördern.

Die vorbereitenden Begegnungen waren nicht nur im Sinne erweiterter Fachkenntnisse von erheblicher Bedeutung. Sie trugen in besonderem Maße zur gegenseitigen Vertrauensbildung als Grundlage für einen konstruktiven Dialog von Mitarbeiterinnen und Mitarbeitern so unterschiedlicher Vollzugssysteme bei. 
Weiterhin hat eine bemerkenswerte Anzahl Beteiligter beider Seiten und Personen mit sehr unterschiedlichen Befugnissen und Zuständigkeiten (Studenten bis Behördenleiter) unmittelbar an den zahlreichen Begegnungen teilgenommen - ein Umstand, der perspektivisch geeignet ist, gewonnene Erkenntnisse Dritten im jeweils eigenen Land $\mathrm{zu}$ vermitteln und - wenn nicht für aktives Mittun - so doch für Akzeptanz und Unterstützung des derzeit laufenden Programms zu werben - trotz bestehender erheblicher Schwierigkeiten, die allerdings im Jahr 2003 verringert werden konnten (s.u.).

\section{Probleme}

Die Herzlichkeit in den Begegnungen und die beiderseits organisatorisch nahezu perfekt gestalteten Begegnungswochen täuschen nicht darüber hinweg, daß es noch erhebliche Schwierigkeiten $\mathrm{zu}$ meistern gilt, wenn sich - zunächst natürlich nur in kleinen, ausgewählten Modellbereichen - ein nachhaltiger Erfolg einstellen soll:

- Die Sprachbarriere - die deutsche Seite ist nicht in der Lage, in russischer Sprache zu kommunizieren, die Russische nicht in deutscher oder englischer Sprache. Die Kommunikation kann nur über Dolmetscherinnen aus dem Hochschulbereich sichergestellt werden, was bei direkten Delegationstreffen gut organisiert werden kann. Es ist allerdings nicht gelungen, eine laufende und dichte Kommunikation über e-mail mit irgendeiner Person aus der Vollzugsverwaltung aufzubauen - wo doch bei drei Partnerbereichen und einer Gebietsverwaltung mindestens vier solcher Kommunikationsbahnen erforderlich wären.

- Die hierarchischen Bedingungen - während in Schleswig-Holstein sich im Laufe dieses Jahres mit Unterstützung der Aufsichtsbehörde ein Projektteam gebildet hat, das weitgehend selbständig Inhalte und Verfahren des knowhow-Transfers entwickeln kann, ist - zumindest aus deutscher Sicht - noch unklar, wer in $\mathrm{Ar}$ changelsk mit welchen konkreten Zielen das Projekt mitgestaltet: Seit unserer Abreise im September 2002 gab es noch keinen unmittelbaren Kontakt mit unseren De- legationspartnern in den drei Einrichtungen. Es scheint, daß eine Person fehlt, die koordiniert und steuert (Dolmetscherkontakte, zur Verfügung gestellte Materialien, Vorschläge, Anfragen u.s.w.) und dabei weitgehend weisungsungebunden agieren kann. Es ist auch unklar, in welchem Rahmen z.B. unsere Partner in den Einrichtungen selbständig experimentieren und gestalten können.

- Kollektivistische Gesellschaftstradition als Grundlage zur Einführung individualisierender Lebenslagengestaltung - die Gestaltung des Vollzuges in Rußland erscheint aus der Sicht der schleswig-holsteinischen Delegation an einer bestimmten Gefangenenzahl (z.B. 100-Mann-Schlafsaal) ausgerichtet zu sein. Soziale Maßnahmen, von denen einige geeignet sind, den Vollzugsalltag erträglicher zu gestalten, Verantwortungsbereitschaft $\mathrm{zu}$ fördern und Kreativität freizusetzen, sind weitgehend Maßnahmen, die die gesamte Wohngruppe einbeziehen. Das Leben erinnert eher an traditionelles Militär, dessen Funktionieren zwangsläufig von der Qualität des Prinzips Befehl und Gehorsam und von einem insgesamt positiven Zustand eines Kollektivs abhängt. Wir haben den Eindruck gewonnen, daß diese Prinzipien in den Köpfen aller dort Beteiligten - Bedienstete wie Gefangene - fest verankert sind. ${ }^{4}$ Die Schwierigkeit besteht darin, vor diesem Hintergrund die Prinzipien und Methoden einer auf das Individuum zugeschnittenen eher partnerschaftlichen Sozialarbeit zu verdeutlichen und experimentell umzusetzen.

- Innovationshemmende Rahmenbedingungen in den betroffenen Behörden - neben dem zuvor beschriebenen grundlegenden Unterschied der bestehenden sozialpädagogischen Konzepte beobachten wir Hemmnisse, die es in unterschiedlicher Ausprägung überall auf der Welt - z.T. auch in Deutschland - gibt. Sie seien ohne weitere Erläuterung hier nur stichwortartig aufgezählt:

- Zahlenverhältnis Sicherheitspersonal/Stations(Erziehungs) personal

- Vorstellung vom »Kriminellen« (Menschenbild) - Vorstellung von Wirkungszusammenhängen zwischen Repression und Verhaltensänderung
- Informations- und Innovationsabneigung (Wer will schon Veränderungen? Man sagte uns, dass in den Gefängnissen und Ministerien vielleicht 5\% der Verantwortlichen das Erfordernis von Veränderungen sehen und diese wünschen)

- $\quad$ Frage des Verständnisses von Grundrechten (z.B. Arrestgebäude, Hunde)

- $\quad$ Fehlende Interventionsund Hilfemöglichkeiten insbesondere durch externe Dienstleistungen.

- Die quantitativen Erfordernisse im Verhältnis zur Zahl der auf beiden Seiten engagiert mitarbeitenden Personen

Nach der Auswertung der Arbeit im Jahr 2002 waren sich alle Beteiligten auf russischer und deutscher Seite über folgendes Grundsatzziel einig:

Es soll erreicht werden, exemplarisch und modellhaft in den zwei beteiligten Vollzugseinrichtugen ein Verfahren individualisierender, lebenslagenklärender Sozialarbeit (Erwachsenenvollzug 42/1) und Methoden partizipativer Gruppensozalarbeit (Jugendkolonie) so zu etablieren, dass deren Chancen, sich selbst fortzuentwickeln und zu verallgemeinern als realistisch erscheinen. Im Ausbildungszentrum müssen zunächst Fortbildungsinhalte und-methoden eingerichtet werden, die geeignet sind, die Entwicklungsarbeit der in den vorgenanten Einrichtungen unmittelbar beteiligten Bediensteten fördernd zu begleiten.

\section{Arbeit und Ergebnisse im Jahr 2003}

Vom 03. bis 18. Mai 2003 besuchte die Delegation russischer Vollzugsmitarbeiterinnen und Mitarbeiter Schleswig-Holstein. Vom 16. - 30. August setzten beide Delegationen ihre Zusammenarbeit in den entsprechenden Einrichtungen in Archangelsk fort. Die Arbeit fand nunmehr im wesentlichen in den Partnereinrichtungen (vgl.o. 2 a) statt:

a) Im Erwachsenenvollzug (Partnerschaft zwischen der Anstalt 42/1 in Archangelsk und der JVA Neumünster) fand während der beiden
Treffen der praktische Einstieg in die individuell lebenslagenklärende Sozialarbeit statt: die beiden Delegationsmitglieder aus Archangelsk hospitierten in den Zugangsabteilungen der Untersuchungshaft und der (geschlossenen) Strafhaft, nachdem sie zuvor schon übersetzte Erhebungs- und Planungsformulare erhalten hatten. Sie nahmen an Erhebungs- (Anamnese-) gesprächen teil, berieten mit den Mitarbeiterinnen über die Inhalte künftiger Vollzugsplanung und nahmen an Vollzugsplankonferenzen teil. Außerdem besuchten sie Dienststellen der sozialen Dienste der Justiz sowie die von Spezialanbietern der Freien Straffälligenhilfe (z.B. Drogen- und Schuldnerberatung). Der Aufenthalt der Mitarbeiterinnen aus SchleswigHolstein in Archangelsk diente dazu, das zuvor Erarbeitete gemeinsam mit den russischen Kollegen in deren Einrichtung umzusetzen. Trotz nicht immer einfacher Bedingungen ${ }^{5}$ gelang es, exemplarisch Vollzugsplanungen durchzuführen und für das in Archangelsk verwendete Gefangenen-Datenblatt ergänzend Elemente der deutschen Vollzugsplanung einzuführen - eine zunächst einrichtungsbezogene Maßnahme, die aber gute Aussichten auf Verallgemeinerung hat

b) Die Delegationsmitglieder der Jugendanstalt Schleswig, Sozialtherapie der JVA Lübeck und der Jugendkolonie Archangelsk verwendeten (neben eingehenden Besichtigungen und Hospitation im sozialtherapeutischen Bereich der JVA Lübeck) die Arbeitszeit in Schleswig-Holstein dazu, Entscheidungsund Konfliktbewältigungsprozesse in einer Jugendwohngruppe der Jugendanstalt zu studieren und daraufhin zu betrachten, welche Elemente bzw. Methoden in Archangelsk erprobt werden könnten.

Eine solche Erprobung fand in der Jugendkolonie auch statt: gemeinsam mit den dort zuständigen Mitarbeitern wurde 4 Gruppen inhaftierter Jugendlicher die folgende Aufgabe ge-

stellt: Sie würden je Gruppe $25 €$ (für russische Verhältnisse eine Menge Geld!) erhalten, wenn sie gemeinsam entscheiden könnten, für welchen Zweck sie es ausgeben wollten. Bedingung allerdings sollte sein, dass der Verwendungszweck allen Gruppen- 
mitgliedern in gleicher Weise zugute kommen sollte. Diese Aufgabe führte in allen vier Gruppen zu realisierbaren Ergebnissen und setzte bei den Jugendlichen wie beim Vollzugspersonal Kreativität und Methoden konstruktiver Entscheidungsfindung frei. Es wurden brauchbare Ideen für die Organisation und Durchführung künftiger gruppenorientierter Jugendsozialarbeit entwickelt 6 . Begünstigend hat sich für die Entwicklung der Zusammenarbeit ausgewirkt, dass der Anstaltsleiter der Jugendkolonie Mitglied der russischen Delegation ist und am Fortschritt von Reformbemühungen in seiner Einrichtung äußerst interessiert ist.

a) In den beiden Aus- und Fortbildungseinrichtungen wurden folgende Arbeitsschritte durchgeführt:

1) Hospitationen der russischen Kollegen im Ausbildungsunterricht in Schleswig-Holstein

2) Planung von Modellunterrichtseinheiten in der Fortbildung russischen Vollzugspersonals

3) Durchführung und Auswertung der Modellunterrichtseinheiten.

Während des Aufenthaltes der russischen Delegation in SchleswigHolstein fanden die Hospitationen wie geplant statt (so z.B. in einem Einführungskurs für Berufsanfänger ein Unterrichtsvormittag im Fach Staats- und Verfassungsrecht). Die Veranstaltung wurde durch die russischen Kollegen mit einer Videokamera aufgezeichnet.

Allerdings ist nicht deutlich geworden, ob und welche Rückschlüsse die russischen Kollegen für die Ausbildung ihres eigenen Nachwuchses aus den Hospitationen gezogen haben und wozu die Videoaufzeichnungen konkret verwendet werden.

Grundlage der weiteren Planung war das neue Handbuch des International Centre for Prison Studies zum menschenrechtsorientierten Vollzugsmanagement, das in englischer und russischer Sprache vorlag. Nach eingehender Erörterung einigten sich beide Seiten darauf, dass die deutschen Delegationsmitglieder in Archangelsk exemplarisch - im Rahmen der Fortbildung aktiven Vollzugspersonals - jeder in jeweils einer beliebigen Zielgruppe 3 - 4 tägige Unterrichtseinheiten zu den Kapiteln 2 (Prison Staff and the Administration of
Prisons) und 3 (Prisoners are Human Beings) durchführen sollten. Ziel sollte sein, zeitgemäße Methoden der Erwachsenenbildung zu erproben und die Wirkung als relevant erachteter Unterrichtsinhalte festzustellen, um daraus verwertbare Erkenntnisse für eine Konzeption der Aus- und Fortbildung am Ausbildungszentrum zu erlangen.

Die Unterrichte fanden in Archangelsk wie vorgesehen statt, allerdings wurde schon in der Vorbereitung deutlich, dass angesichts der Absicht, Inhalte durch die jeweilige Kursgruppe selbst erarbeiten zu lassen (statt Frontalunterricht), in der zur Verfügung stehenden Zeit lediglich ein Kapitel (Prison Staff) Gegenstand des Unterrichts sein konnte.

Methoden und Inhalt fanden bei den Kursteilnehmern Interesse und Zustimmung, allerdings läßt sich nach diesem »Probelauf « und angesichts des derzeitigen Entwicklungsstandes der angestrebten Veränderung/Ergänzung des Aus- und Fortbildungsbetriebes in Archangelsk noch keine klare konzeptionelle Zielrichtung für Innovationen erkennen. Dies liegt unseres Erachtens u.a. daran, dass Schulleitung und Lehrkörper trotz der Vorbereitung in SchleswigHolstein vermutlich nicht klar war, was durch die Lehrproben in Archangelsk erreicht werden sollte und wie Erwachsenenbildung inhaltlich und methodisch entwickelt werden sollte. Hier muß überlegt werden, ob der Lehrkörper oder Teile davon selbst eine Fortbildung zum Thema »Management von Aus- und Fortbildung « erhalten müßten, bevor konkret weitergeplant wird.

\section{Ausblick und weitere Planung}

Der Leiter der Vollzugsverwaltung des Gebietes Archangelsk, General Malov, stellte während der gemeinsamen Auswertung des bisher Erreichten in seiner Stellungnahme fest, dass nicht nur im Gebiet Archangelsk sondern in der gesamten Russischen Föderation Menschenrechte und soziale Fürsorge zunehmend die Innovationsbemühungen im Strafvollzug kennzeichneten. Das Projekt der vollzuglichen Zusammenarbeit zwischen dem Gebiet Archangelsk und dem Land Schleswig-Holstein liege daher hinsichtlich seiner Zielsetzung und der bisher erreichten Ergebnisse auf der Linie allgemein anerkannter Erfordernisse. Insgesamt hob er die durch die Delegationen geleistete erfolgreiche Arbeit hervor und äußerte den Wunsch dass diese Arbeit nicht nur über 2004 fortgesetzt sondern intensiviert werden sollte.

Gemeinsam betonten die Delegationen vor dem Hintergrund ihrer bisherigen Erfahrungen, dass Veränderungen trotz noch zum Teil alter Strukturen, Vorschriften und Denkweisen möglich seien. Dies hänge im wesentlichen von den beteiligten Personen ab. Sie mahnten an, dass die Leitung der Vollzugsverwaltung eine handlungsfähige Projektgruppe für Innovationen schaffen müsse. Sie regten weiterhin an, dass die Vollzugsverwaltung in ein Aus- und Fortbildungskonzept auch die PomorenUniversität mit ihrer Erfahrung einbinden sollte, zumal in einem Teilbereich schon eine Spezialisierung der universitären Ausbildung auf Straffälligenhilfe erfolgt sei.

Folgende Programmerweiterung wird von beiden Seiten angestrebt:

a) Verlängerung der Delegationstreffen auf zwei Arbeitswochen (2x5 Arbeitstage), da dies relativ überschaubare Mehrkosten verursachen würde. Es wird darauf hingewiesen, dass angesichts der bereits geleisteten konkreten intensiven Arbeit noch ein erhebliches Pensum sichtbar geworden ist und zudem (vgl. o.) in allen drei Arbeitsbereichen die Zeit nicht ausreichte, so dass auf Urlaubstage zurückgegriffen werden musste.

b) Ein dringlicher Innovationsbedarf besteht im Hinblick auf die Humanisierung der Untersuchungshaft. Aus diesem Grund wird angestrebt, als vierten Entwicklungsbereich die Untersuchungshaft zusätzlich in das Projekt zu nehmen und beide Delegationen für diese Aufgabe um jeweils zwei Mitarbeiter zu erweitern.

Abschließend ist festzustellen, dass die konkrete auf einen überschaubaren aber wichtigen Bereich der vollzuglichen Arbeit beschränkte Partnerschaft erfolgversprechend ist. Wesentlich dafür ist auch, dass die Entwicklungsarbeit dort stattfindet, wo auch der Vollzug stattfindet, nämlich in den Vollzugseinrichtungen und mit den Menschen, die täglich die Arbeit mit den Gefangenen leisten.

Trotz der oben beschriebenen fortdauernden hemmenden Bedingungen (die sich auch ohne weiteres nicht beseitigen lassen) und der geringen Anzahl der permanent Beteiligten, lässt sich sagen, dass schon jetzt von einer gewissen Nachhaltigkeit des bisher Erreichten auszugehen ist.

Allerdings legt der Umfang dessen, was quantitativ, organisatorisch und inhaltlich noch zu bewegen ist, nahe, die Arbeit über 2004 hinaus fortzusetzen und auszuweiten. Dies gilt zum einen für den Justizvollzug selbst. Ein weiteres wesentliches Stichwort für den Bereich der Sozialarbeit ist der Aufbau einer Infrastruktur für eine auch außerhalb des Vollzuges angesiedelte Straffälligenhilfe, da ohne sie vollzugliche Sozialarbeit weitgehend wirkungslos bleiben muß

Wolfgang Gottschalk (Projektkoordinator für Auslandsprojekte der schleswigholsteinischen Vollzugsverwaltung)

\section{Anmerkungen}

1 Mit Hilfe des AHP wurde der Aufbau eines Studiengangs »Sozialarbeit und Sozialpädagogik « über Partnerschaften der Fachhochschulen Kiel und Emden mit der Pomoren-Universität in Archangelsk ermöglicht. In diesen Aufbau wurden und werden unterschiedliche Praxisfelder der Sozialarbeit (z.B. Behinderten-, Altenund Jugendhilfe) einbezogen. Dazu gehörte in den letzten Jahren auch die Straffälligenhilfe insbesondere der Justizvollzug und die Bewährungshilfe.

2 Vgl. ausführlichen Bericht der schleswig-holsteinischen Vollzugsverwaltung v. 22.06.2002

3 vgl. Bericht der schleswig-holsteinischen Vollzugsverwaltung vom 18.10.2002

4 In der Anstalt 42/5 konnten wir von diesen Prinzipien erfreuliche Abweichungen registrieren, so u.a. eine Wohnung für einen (langstrafigen) Gefangenen zu dessen Vorbereitung auf die jeweils in ca. 4 Wochen bevorstehende Entlassung

5 Auch hier wird ein detaillierter Bericht demnächst vorliegen

6 hierzu werden die Beteiligten einen detaillierten Bericht abgeben, der bei Interesse im MJF Schlesw.Holst. angefordert werden kann.

7 Coyle, Andrew, »A Human Rights Approach to Prison Management $«$, London 2002 